\title{
Experiências brasileiras sobre participação de usuários e familiares na pesquisa em saúde mental
}

\author{
Brazilian experiences in the participation of users \\ and family members in mental health research
}

Rodrigo Fernando Presotto ${ }^{1}$

Marília Silveira ${ }^{2}$

Pedro Gabriel Godinho Delgado ${ }^{3}$

Eduardo Mourão Vasconcelos ${ }^{4}$

${ }^{1}$ Departamento de Medicina Preventiva e Social,

Faculdade de Ciências

Médicas, Universidade

Estadual de Campinas. R.

Tessália Vieira de Camargo,

Cidade Universitária.

13.083-887 Campinas SP

rodrigopresotto@yahoo.com.br

${ }^{2}$ Universidade Federal do

Rio Grande do Sul.

${ }^{3}$ Nuppsam Núcleo de

Pesquisas em Políticas

Públicas de Saúde Mental,

Instituto de Psiquiatria,

Universidade Federal do

Rio de Janeiro.

${ }^{4}$ Departamento de Métodos

e Técnicas, Escola de

Serviço Social,

Universidade Federal do

Rio de Janeiro.

\begin{abstract}
In this paper the authors describe and contextualize the participation of users and family members in mental health research in Brazil, addressing the recent tradition of the experiences of recovery and empowerment to define the analysis of some Brazilian experiences of evaluative research and intervention projects, which count on these social actors to act as researchers. The experiences of Self-Help Groups and the Guide to Autonomous Medication Management are described briefly, in order to analyze the limits and possibilities of participation of users and their family members in research, which is still incipient and sporadic in the Brazilian reality. The authors also recommend the creation of an agenda in public health policy that encourages this participation.
\end{abstract}

Key words Citizen participation, Consumer participation, Social participation, Mental health, Empowerment, Recovery
Resumo Neste artigo os autores fazem a contextualização e a descrição da participação de usuários e familiares em pesquisas em saúde mental no Brasil, abordando a tradição recente das experiências de recuperação (recovery) e empoderamento (empowerment), para delimitar a análise de algumas experiências nacionais avaliativas e projetos de intervenção que contam com estes atores sociais como pesquisadores. São relatadas, sucintamente, as experiências de Grupos de Ajuda Mútua e Guia da Gestão Autônoma de Medicação, com o objetivo de analisar os limites e as possibilidades da participação de usuários e familiares na pesquisa, ainda incipiente e pontual na realidade brasileira. Os autores recomendam a construção de uma agenda na política pública de saúde que estimule esta participação.

Palavras-chave Participação cidadã, Participação comunitária, Participação social, Saúde mental, Empoderamento, Recovery 


\section{Introdução}

No presente trabalho, objetivamos descrever algumas direções brasileiras de desenvolvimento de pesquisas e projetos interventivos que levam em consideração direta ou indiretamente a perspectiva participativa ou produtora de autonomia de usuários e familiares. Como indicado em artigo anterior ${ }^{1}$, a partir da segunda metade da década de 2000, ativistas, gestores, pesquisadores e profissionais do campo da saúde mental no Brasil passaram a ampliar seus contatos internacionais e a abrir iniciativas de intercâmbio e de pesquisas conjuntas com países anglo-saxônicos, em processo explícito de valorização da perspectiva do empoderamento (empowerment).

Nesta área, podemos notar pelo menos duas direções básicas em nosso país: uma tradição de pesquisa avaliativa de programas e serviços em saúde e saúde mental, com base em formas complexas de participação dos usuários do SUS; e projetos-piloto de experimentação e sistematização de dispositivos de empoderamento em saúde mental, integrados em pesquisas ou com estratégias participativas de produção de conhecimento ou de avaliação.

\section{Usuários como participantes de equipes de pesquisa no campo da saúde mental}

O tema da participação do usuário aproxima-se das formulações presentes na coalização de movimentos, coletivos e pesquisadores, reunidos sob denominações diversas, mantendo especificidades relevantes, e que defendem uma mudança de paradigma na atenção psiquiátrica, de modo a afirmar a autonomia do usuário como um norte ético, o território e o cotidiano como lugares e tempos privilegiados para o cuidado, e a construção de formas autônomas de superação das limitações impostas pelo sofrimento mental. Tais movimentos valem-se das contribuições dos grupos de ajuda mútua, do empoderamento, da recuperação ou restabelecimento (recovery) e da reabilitação psicossocial.

Para este campo multifacetado, a experiência de vida de usuários e familiares e sua autonomia são direções tanto éticas quanto metodológicas; assim, o tema da participação em pesquisa assume relevância e se torna um desafio intrínseco e obrigatório.

A pesquisa no campo prático e teórico do recovery deve incluir necessariamente a participação de usuários como protagonistas na produção de conhecimento. Segundo Slade ${ }^{2}$, reco- very é um caminho, um processo, e vincula-se a uma visão da medicina baseada em valores (e não apenas em provas e evidências científicas). Para tornar este movimento uma realidade, é preciso desenvolver caminhos de investigação e métodos de validação capazes de avaliar com acuidade os dispositivos baseados em tal perspectiva (recovery-focussed mental health services), no âmbito internacional ${ }^{3}$. Farkas e Anthony, pesquisadores importantes do campo do recovery ${ }^{4}$, destacam que o desenvolvimento de conhecimento, atitudes e competências dos usuários para lidar com os problemas do sofrimento deve ser uma busca sistemática na pesquisa e, portanto, constitui um desafio para a participação direta do usuário na equipe de pesquisa.

Simpson e House ${ }^{5}$ realizam uma revisão sistemática sobre o envolvimento de usuários na avaliação de serviços de saúde mental, identificando que, em geral, muito poucos usuários estavam de fato incorporados nos projetos avaliativos. Apesar de tais limitações, cresce o reconhecimento da importância da sua participação, seja na etapa de planejamento, como na de avaliação dos serviços de saúde mental ${ }^{6}$. Serviços dirigidos por usuários são objeto de pesquisa, comparados a serviços convencionais ${ }^{7}$, e constituem um dos caminhos para a busca de participação efetiva de usuários na produção de conhecimento no campo da atenção psicossocial. Desafios metodológicos para a pesquisa sobre grupos de apoio mútuo em saúde mental são abordados por Goldstrom et al. ${ }^{8}$, apontando para a necessária participação dos usuários em tais avaliações. $\mathrm{O}$ tema da participação de usuários na pesquisa, ainda incipiente na reforma psiquiátrica brasileira, está presente de forma intensa na pesquisa internacional, no campo delimitado pelos marcos teóricos da reabilitação psicossocial, empoderamento e recovery. No campo de investigações da saúde pública este desafio já vinha se colocando desde o final da década de $1990^{\circ}$. Na literatura internacional encontramos a efetiva participação de usuários de saúde mental nos grupos de investigação. Tais estudos identificam os desafios metodológicos, éticos e teóricos decorrentes desta empreitada e suas contribuições podem ajudar a nortear este tipo de trabalho no Brasil. Algumas pesquisas, como as que traremos a seguir, dão mostras do desenvolvimento deste tema no país. Além das pesquisas, outras experiências, como a participação de usuários no ensino da psicopatologia ${ }^{10}$, vêm se construindo há mais de cinco anos no ambiente da universidade pública. 
Por que usuários (e familiares) devem participar das pesquisas em saúde pública?

A saúde pública brasileira, na conformação atual do SUS, data da recente redemocratização do país, tendo pouco mais de vinte anos. Embora a participação do usuário, na forma do chamado "controle social", seja uma das marcas importantes e valiosas do novo sistema, a agenda do SUS (e do campo acadêmico da saúde pública) não incorpora ainda o usuário como coprotagonista da produção do conhecimento em saúde.

Entretanto, a implicação dos usuários na pesquisa em saúde é um dos temas cruciais do debate da saúde pública na última década, especialmente em países como o Reino Unido, Canadá e Austrália, além de outros. Uma revisão sobre o tema ${ }^{11}$, no sistema de saúde inglês (NHS - National Health System), discute os diversos níveis de participação dos usuários na pesquisa em saúde, especialmente no âmbito de avaliação de procedimentos e serviços, e aponta as objeções, de natureza ideológica, que esta participação suscita, especialmente entre profissionais de saúde e pesquisadores. Segundo algumas dessas objeções, a participação de usuários, recomendada pelo NHS desde 1999, seria resultado de uma indesejável "politização" da saúde pública inglesa, não sendo "baseada em evidências",

A participação do usuário não é aqui entendida apenas como "sujeito de pesquisa", conforme definido nas normas brasileiras de ética em pesquisa com seres humanos ${ }^{12}$, mas como integrante da equipe de investigação, o que traz desafios importantes a serem ainda enfrentados, como a própria avaliação cuidadosa e sistemática desta contribuição. O que importa ressaltar é que, embora vista como "paternalista" por muitos especialistas, a incorporação efetiva de usuários na pesquisa em saúde é parte integrante da agenda do sistema de saúde inglês. Entre outros atores que defendem esta participação é relevante citar o Fórum de Usuários de Saúde da Austrália, cujos objetivos incluem a participação sistemática do público na construção das pesquisas em saúde ${ }^{13}$. Esta é, portanto, uma questão para a agenda internacional da pesquisa em saúde pública, não apenas para a saúde mental.

A partir daqui apresentamos algumas experiências brasileiras especialmente direcionadas às práticas do empoderamento e do recovery, nas quais se inserem os autores deste artigo.

\section{Projeto de uma metodologia para grupos} de ajuda e suporte mútuos em saúde mental

No Brasil já existe uma relativa diversidade de projetos e ações de suporte mútuo, defesa dos direitos, mudança na cultura e militância social, a maioria estimulados pelo movimento antimanicomial e geralmente realizados de forma integrada com os serviços de atenção psicossocial. Entretanto, estas experiências, próprias do movimento de usuários e familiares, apresentam enorme fragilidade, existindo poucas iniciativas de ajuda mútua diferenciadas dos modelos clássicos da tradição dos 12 passos, oriundos dos Alcóolicos Anônimos ${ }^{14}$.

Foram experimentadas e sistematizadas metodologias diversificadas de empoderamento, particularmente de narrativas pessoais de usuários, de vida e convivência com o transtorno ${ }^{15}$, e de grupos de ajuda e suporte mútuos no campo da saúde mental. Nesta última área, iniciou-se em 2008 uma capacitação para facilitadores usuários, familiares e apoiadores de grupos-piloto de ajuda e suporte mútuos no Rio de Janeiro. No ano seguinte passou a contar com apoio do Ministério da Saúde e desenvolveu-se em outras regiões do Brasil. Os produtos dessa pesquisa foram sistematizados numa Cartilha e Manual de Ajuda e Suporte Mútuos em Saúde Mental, que já se encontram disponíveis na forma digital ${ }^{16}$.

Boa parte das ciências biomédicas, clínicas e comportamentais não têm muito que oferecer, de forma satisfatória, diante das complexas questões da emoção, da dor, do desespero, da angústia moral e da perda de sentido de vida associadas ao transtorno mental. No enfrentamento destes dilemas e desafios concretos do cotidiano com o sofrimento psíquico, os grupos de ajuda mútua valorizam e promovem a troca da experiência acumulada pelos usuários e familiares. Com base em suas próprias referências culturais e recursos disponíveis, além de criar um ambiente de acolhimento para as vivências marcadas pelo sofrimento, geram cumplicidade e apoio mútuo. Quanto à dinâmica de funcionamento, a característica principal dos grupos é serem facilitados pelos próprios usuários e familiares em estágio mais avançado de recuperação e de convivência com o transtorno mental. Tais atores contam com uma capacitação específica e provisão de assessoria e supervisão por profissionais devidamente capacitados. O projeto trabalha com grupos distintos para usuários e para familiares. Em todas as demais atividades, como coordenação e supervisão, projetos de suporte mútuo, defesa de direitos e mili- 
tância social, usuários e familiares atuam juntos. O grupo deve ser facilitado sempre em duplas, por pessoas previamente capacitadas, para garantir mais estabilidade e segurança nas atividades, e a possibilidade de um dos facilitadores atuar eventualmente com formas mais individualizadas de apoio durante as reuniões. Os encontros têm uma duração média de 3 horas e há vários tipos previstos de reunião, mas a modalidade mais comum é a de troca livre de experiências. Os grupos, em geral realizados em espaços externos aos serviços, visam primordialmente que usuários e familiares busquem caminhos fora dos serviços assistenciais, vivenciando o território, ocupando locais na comunidade que permitam realizar uma reunião com privacidade: escolas públicas, bibliotecas, abrigos, associações de usuários e familiares, associações de moradores, sindicatos, centros de referência de assistência social (CRAS) etc. A integração com a rede de atenção primária à saúde é também fortemente encorajada. Os grupos podem ser voluntários, quando inseridos nas associações de usuários e familiares. Entretanto, a inserção na rede de saúde, saúde mental e assistência social requer o pagamento de bolsas de trabalho e outras formas de suporte para os facilitadores, e no futuro espera-se que possam evoluir para contratos formais de trabalho.

Os desafios enfrentados pelos grupos são vários, do ponto de vista teórico, metodológico e prático-operativo, como fica expresso no manual elaborado durante a experimentação e sistematização do projeto. No entanto, a avaliação mais geral da metodologia nestes quatro anos de projeto-piloto mostra que o dispositivo é viável, seguro e produz efeitos bastante positivos para a vida mais ampla e para o quadro de saúde mental dos participantes. Independente de serem ou não usuários regulares de outros serviços de saúde, bem como produzem efeitos na própria dinâmica interna dos Centros de Atenção Psicossocial (CAPS) nos quais o projeto foi inserido. Os grupos fomentam a participação no sistema de saúde e saúde mental, e em outras instâncias do movimento de usuários e familiares e do movimento antimanicomial.

Embora vise o empoderamento de usuários e familiares, este projeto pode ser classificado como pesquisa participante e interventiva, de cunho qualitativo e inteiramente controlada pela equipe de pesquisadores ${ }^{17}$. Mas conta com inúmeros dispositivos de expressão e escuta permanente dos demais atores sociais envolvidos e de revalidação das sistematizações regulares feitas pelos pesquisadores. Alguns princípios ético-políticos, teóri- co-conceituais e metodológicos que orientaram a implantação e a sistematização da experiência são que o processo de produção de conhecimento assume a complexidade e a multidimensionalidade do real, em uma práxis com maior nível de incerteza e riscos. Conta com cronogramas mais alongados que exigem um rigor epistemológico e metodológico ${ }^{18}$. A revisão bibliográfica é tomada como dispositivo interdisciplinar permanente. $\mathrm{O}$ desenvolvimento de uma base de sustentação interteórica complexa e não linear permite não só construir elementos de contextualização e comparação, mas também forjar uma sustentação teórico-conceitual mais rica. São necessários cuidados na apropriação transnacional crítica de abordagens e experiências internacionais, pois abordagens e dispositivos originados em outros países requerem um enorme cuidado com as diferenças sociais, econômicas, política e culturais envolvidas nestas práticas. Os métodos de pesquisa participante, interventiva e qualitativa, têm os resultados convertidos em acréscimos regulares às inúmeras versões da cartilha e ao manual. Estes depois são testados e revalidados em novas conversas com os participantes, nas supervisões e nas próprias reuniões de grupo, nos seminários em vários locais do país, e em leituras sistemáticas. Assim, diferentes tipos de orientação e dispositivos grupais são colocados à prova e discutidos com os participantes. Com os desafios e problemas que emergem, estratégias alternativas de ação são devidamente examinados e avaliados à medida que surgem. Os resultados deste processo de testagem e revalidação realizada ao longo dos vários anos de experimentação da metodologia foram formatados para serem incluídos nas diversas seções da cartilha e do manual. Assim, buscou-se produzir textos em estilo mais simples possível, em diferentes seções de complexidade crescente, accessíveis e adequados para os diversos tipos de leitores. Trabalhou-se, portanto, com o princípio da saturação, pelo qual se avalia o processo de maturação da metodologia, do texto e da pesquisa pelo número decrescente de acréscimos e correções necessárias, só permitindo sua publicação final quando estas tendem a ficar residuais. Realizou-se também uma divulgação massiva por meio digital, para ampliar o acesso à cartilha e ao manual para o universo de pessoas que potencialmente poderiam contribuir para a avaliação do material. No processo, estava prevista a redação e a divulgação de várias versões preliminares da cartilha e do manual na forma de apostila e texto digital, para uso, testagem, e saturação em capacitações, seminários, supervisões e nos 
grupos, antes da versão e publicação final, bem como a avaliação mais sistemática por leitores qualificados, incluindo contatos internacionais.

Outro exemplo, no cenário brasileiro, de participação de usuários em pesquisa (neste caso, familiares de pacientes portadores de transtornos mentais graves em atendimento nos CAPS da região metropolitana do Rio de Janeiro), é o projeto Familiares Parceiros do Cuidado, iniciado em 2011, na UFRJ. Seguindo igualmente o percurso metodológico de pesquisa interventiva, apoiada nas referências conceituais do recovery, produção de autonomia e grupos de ajuda mútua, tem estimulado a participação direta dos familiares na análise do material empírico da pesquisa $^{18}$.

\section{Pesquisa avaliativa de saúde mental: instrumentos para a qualificação da utilização de psicofármacos e formação de recursos humanos - GAM-BR}

Esta pesquisa é um estudo avaliativo e participativo, voltado para CAPS de três municípios brasileiros: Rio de Janeiro, Novo Hamburgo (RS) e Campinas, e envolveu quatro universidades: a UNICAMP, UFRGS, UFF e a UFRJ. Este estudo teve como objetivos: traduzir, adaptar e testar em CAPS destas cidades o Guia Pessoal de Gestão Autônoma da Medicação (Guia GAM) ${ }^{19}$. Este instrumento foi desenvolvido no Canadá a partir de experiências de gestão autônoma da medicação desde 1993, pela Associação de Recursos Alternativos de Saúde Mental de Quebec, com a colaboração da associação dos grupos de intervenção em defesa dos direitos em saúde mental de Quebec (AGIDD-SMQ) e pesquisadores da Equipe de Pesquisa Ação em Saúde Mental e Cultura (ÉRASME) - e buscou avaliar o impacto desse instrumento na formação de profissionais de saúde mental (psiquiatras e profissionais não médicos).

A justificativa para sua realização no Brasil aliou-se ao fato de que a iniciativa canadense buscava implantar um processo de questionamento, experimentação e pesquisa para interrogar o lugar da medicação na vida das pessoas, seu papel nas práticas profissionais e sua penetração e percepção no conjunto da sociedade ${ }^{20}$. A abertura desses espaços de diálogo permitiu enriquecer o olhar sobre as práticas em saúde mental e elaborar outra abordagem de intervenção denominada Gestão Autônoma da Medicação (GAM).

Esta abordagem era uma estratégia possível para tentar lidar com um problema revelado por algumas pesquisas já existentes no Brasil e no mundo que apontam o aumento significativo no consumo de psicofármacos por parte da população ${ }^{21-25}$. Pessoas portadoras de transtornos mentais usuárias de serviços públicos de saúde e saúde mental ou não, questão que permanece ainda hoje pouco problematizada pela Reforma Psiquiátrica Brasileira.

Para efetivação e aposta nesta possível estratégia, ainda restava um importante passo a ser dado. Não bastava somente traduzir o guia GAM, pois ele não havia sido devidamente testado e adaptado à realidade brasileira. O conteúdo do guia GAM canadense apresentava diferenças em relação à cultura e à realidade brasileiras, às formas como são promovidas as ações de reabilitação e assistência em saúde mental no Brasil e às experiências e vivências dos brasileiros portadores de transtornos mentais.

Ao longo da pesquisa aconteceram grupos de intervenção (GI), que contaram com a participação de usuários, profissionais de saúde e estudantes de pós-graduação, considerados atores ou grupos sociais de extrema relevância para ser, em conjunto com a equipe de pesquisadores, responsáveis pela testagem e, ao final do processo, adaptação do Guia GAM à realidade brasileira. Esses eram os grupos sociais de interesse diretamente envolvidos no contexto pesquisado; conhecer seu ponto de vista era pré-condição para a compreensão da realidade estudada e para a construção de conhecimento condizente com a realidade objetiva vivenciada pelos sujeitos. $\mathrm{O}$ estudo começou a ser realizado, no Brasil, em 2009 com financiamento do CNPq.

Para a coleta de dados foram realizados antes do início dos GI e após seu encerramento, grupos focais (GF) e entrevistas com os atores de cada um dos diferentes grupos sociais de interesse - usuários, familiares, profissionais, residentes e gestores - com intuito de avaliar com cada um destes o processo vivenciado durante as reuniões. Depois da realização dos GF foram construídas narrativas posteriormente apresentadas aos participantes para validação.

Os critérios para participação dos usuários na pesquisa eram a manifestação de interesse pelo tema, não ser uma pessoa com deficiência intelectual e fazer uso da medicação psiquiátrica há pelo menos 12 meses. Alguns participantes foram incluídos a partir de sugestão das equipes, outros quiseram participar por conta própria. Os profissionais dos CAPS que participaram não podiam ser referência no serviço para os usuários pesquisados ou, no caso dos psiquiatras, serem os prescritores da medicação daqueles que 
compunham os grupos. A intenção era não colocar os grupos no lugar do de medicação do serviço. Outra aposta era de que este profissional pudesse ser um articulador e porta-voz, dentro do CAPS, de reflexões relativas à GAM com os profissionais que eram referências dos usuários integrantes da pesquisa, funcionando assim como mais um elemento para pensar o projeto terapêutico individual destes.

Para muitos usuários "os GI constituíram-se como espaço de encontro para as discussões coletivas acerca dos conteúdos a serem abordados pelo Guia GAM"26. Neste sentido, transformaram-se gradativamente em espaços proporcionadores de empoderamento no que diz respeito ao tema da experiência da medicação e também com relação a algo não previsto inicialmente nos objetivos da pesquisa: a temática dos direitos dos usuários de saúde mental.

Em 2010, a pesquisa GAM-BR fez articulação com a Aliança de Pesquisa Universidade Comunidade Internacional (ARUCI). A ARUCI é uma aliança de pesquisa, iniciada no Canadá em uma ação conjunta que envolve dois órgãos daquele país: o Conselho de Pesquisas em Ciências Humanas e o International Development Research Centre (Centro Internacional para o Desenvolvimento de Pesquisas - IDRC, órgão federal canadense de fomento à pesquisa e projetos de caráter inovador). A ARUCI tem como intuito incentivar o desenvolvimento de projetos de pesquisas inovadores, produção de conhecimento sobre questões de vital importância para o desenvolvimento social, econômico e cultural do Canadá e de países em desenvolvimento. A parceria estabelecida entre os representantes brasileiros e canadenses na ARUCI foi nomeada ARUCI - Saúde Mental e Cidadania (ARUCI-SMC). Os agentes responsáveis e, agora, interlocutores nesta parceria foram: as universidades brasileiras envolvidas na pesquisa GAM-BR, a Université de Montreal, a Associação de Pessoas Usuárias de Serviços de Saúde Mental da Região de Québec (APUR) e a Associação Florescendo a Vida de Familiares, Amigos e Usuários dos Serviços de Saúde Mental de Campinas (AFLORE).

Para efetivação desta aliança foi preconizado que os projetos desenvolvidos buscariam a integração entre o conhecimento concebido nas universidades e aquele produzido na comunidade, em movimentos e espaços de trocas sociais, valorizando sua construção conjunta. Visa uma produção engajada com a realidade social; que é fruto de intervenção e investigação e que almeja produzir conhecimento implicado com questões destas diversas ordens elencadas, com o intuito de encontrar maneiras de favorecer o desenvolvimento social, econômico e cultural das comunidades ou grupos comunitários beneficiados pelo programa.

Presotto $^{27}$, em estudo realizado sobre a participação de usuários de serviços de saúde mental em pesquisas desenvolvidas por um dos grupos responsáveis pela pesquisa GAM-BR e pelo desenvolvimento da ARUCI-SMC, aponta o empoderamento e o recovery vivenciados pelos usuários, ao longo de sua participação nas atividades da pesquisa GAM-BR, de ações desencadeadas em decorrência deste processo vivido e em projetos propostos e desenvolvidos pelos mesmos na ARUCI-SMC. Este estudo apontou que a participação dos usuários, de forma geral, trouxe benefícios individuais à maior parte deles, conforme relatos dos mesmos, durante as atividades realizadas nos diferentes campos onde foram desenvolvidas as atividades da pesquisa GAM-BR. Benefícios individuais, pois para muitos a experiência de participação nestas atividades gerou mais ganhos aplicados à esfera do tratamento e melhora na qualidade de vida, sem que houvesse uma ampliação deste conhecimento adquirido para outros âmbitos de sua participação social, como em espaços de atividades e movimentos sociais mais complexos. Contudo, para um número significativo de usuários, alguns membros de associação de usuários e familiares e outros sem histórico de militância, a participação nestas atividades representou viver experiências que contribuíram para que eles passassem a agir com maior apropriação de sua condição de cidadãos frente a situações nas quais, anteriormente, sem estarem participando de algum tipo de intervenção que proporcionasse outros recursos, não agiriam como tal, revelando o processo de empoderamento pelo qual passaram. Os relatos trazidos pelos usuários demonstraram também estratégias que foram ou se tornaram eficazes para o reconhecimento do recovery vivido pelos próprios sujeitos ao longo de suas vidas ou proporcionado por sua participação nas atividades desencadeadas a partir da pesquisa GAM-BR e ARUCI-SMC.

Dentre as principais contribuições sentidas e relatadas pelos usuários estão: aumento de entendimento e autonomia com relação ao transtorno mental e seu tratamento, quando comparado ao momento inicial antes dos grupos; ampliação da compreensão com relação à função da terapêutica medicamentosa, o uso racional da medicação psiquiátrica e a importância das ou- 
tras estratégias e formas de cuidado, de promoção à saúde e de recovery utilizados e valorizados pelos próprios usuários para a sua saúde mental; valorização do espaço coletivo como proporcionador de trocas entre os próprios usuários; importância da horizontalidade nas relações estabelecidas entre todos os envolvidos nas atividades de pesquisa - profissionais, pesquisadores, familiares e outros usuários - o que contribuiu para que os usuários se apropriassem mais ainda de sua condição de sujeitos de direito nas diversas interfaces de sua vida social; aumento de compreensão sobre cidadania e os direitos dos usuários do SUS e da Saúde Mental. A participação nas pesquisas e nas atividades da ARUCISMC proporcionou o primeiro contato para conhecimento de direitos enquanto usuários do SUS e pessoas portadoras de transtornos mentais, para quem desconhecia tais direitos, e aprofundamento e esclarecimento para quem já tinha conhecimento e era militante de movimentos sociais ou membros de conselhos do controle social do SUS; aumento da compreensão, do poder contratual e da capacidade de enfrentamento dos usuários acerca da temática e de situações reais de preconceito e do estigma vividos pelos próprios, enquanto pessoas portadoras de transtornos mentais em sociedade; valorização do processo de construção compartilhada do conhecimento entre pesquisadores, usuários e profissionais de saúde. Salientado por muitos usuários como algo representativo e inédito em suas vidas, e extremamente motivador e fortalecedor de autoestima, por estarem inseridos neste processo; alguns usuários, por conta própria e motivados pela experiência que vivenciaram na pesquisa GAM-BR, referiram motivação para prosseguir, a partir da experiência que lhes foi proporcionada, agora como multiplicadores do conhecimento, no papel de coordenadores de grupo, almejando conduzir projetos de sua própria autoria ou de continuidade daquele do GAM.

Parte dos usuários, ao final da pesquisa GAM-BR, integrou-se à ARUCI-SMC e alguns propuseram projetos que foram contemplados em edital lançado pela aliança que subsidiou dois projetos de intervenção de caráter comunitário e cidadão. O "Guia do Usuário da Saúde Mental (GUSM)", proposto pela AFLORE, e o "Conquista de Cidadania: Como se constrói a cidadania?”, proposto por uma usuária que participou da pesquisa GAM-BR e é relatado a seguir.

O projeto "Conquista de Cidadania: como se constrói a cidadania?”, visava à realização de um grupo de intervenção coordenado por dois usuá- rios para leitura do Guia GAM. A aprovação do projeto no edital permitiu o pagamento dos coordenadores do grupo pela atividade, bem como passagens aos participantes e o lanche oferecido nos encontros. Assim, a participação desses dois usuários coordenadores caracterizou-se efetivamente como um trabalho. Além deles, dez outros usuários do mesmo CAPS participavam do grupo junto com um trabalhador do serviço e um pesquisador. Entretanto, a função do pesquisador e do trabalhador ali era de acompanhar o grupo e auxiliar em algum manejo quando necessário, mas de forma alguma, tomar o lugar de coordenação.

Dessa experiência destaca-se o efeito da cogestão, pois o grupo de usuários movimentou-se para auxiliar os coordenadores, não demandando dos pesquisadores ou dos trabalhadores a função de coordenação. Neste grupo um dos usuários coordenadores era um ouvidor de vozes e alternava-se entre escutar as vozes que só ele ouvia e escutar as vozes das pessoas no grupo. O grupo acolheu o adoecimento da outra coordenadora e auxiliou esse usuário na coordenação do grupo. Por trás dos diagnósticos que haviam recebido e pelos quais se identificavam, os usuários tinham histórias, queriam contar as histórias de seu adoecimento e da experiência de utilizar medicamentos. Neste grupo se viam cenas, que poderiam ser classificadas como delírio por qualquer profissional, serem acolhidas pelo grupo como um traço qualquer de personalidade, com o qual todos podiam se identificar; viam-se alguns usuários adoecerem e se ausentarem e depois retornarem e serem acolhidos pelo grupo. A partir deste projeto, os coordenadores do grupo foram convidados para as reuniões de pesquisa na universidade, nas quais eles podiam fortalecer o vínculo com os pesquisadores, elaborarem as experiências de coordenação e vivenciarem outro lugar no mundo.

O fato de os pesquisadores estarem ao lado dos usuários, como colegas de trabalho, permitiu outra relação e uma aposta de que o usuário pode trabalhar ao lado do pesquisador e desenvolver atividades de pesquisa. O pesquisador não precisa ocupar-se em diagnosticá-lo a partir de seus sintomas, posto que essa não é sua função ali. Permitiu descobrir habilidades dos usuários invisibilizadas no serviço de saúde e uma mudança de lugar provocadora de movimentos na direção do sujeito, para tomar posse de sua própria história como também movimentar-se em direção à cidadania e à inserção social. A partir dessa experiência de coordenação de grupo, esses usuários se tornaram pesquisadores no projeto, 
e essa nomeação foi de uma valia inestimável para eles, que desde o início do adoecimento e do tratamento no CAPS, tinham apenas um lugar no mundo: o de usuário de saúde mental. E a partir dali passaram a ser chamados e a se apresentar como pesquisadores da GAM.

\section{Discussão}

A participação de usuários em projetos de pesquisa em saúde mental é uma experiência recente, tendo surgido na bibliografia internacional especializada nos últimos 20 anos. Configura-se como uma tendência que vem se afirmando, especialmente no Reino Unido, Canadá, Austrália e Estados Unidos. Não se trata aqui apenas da participação, como protagonistas, em intervenções destinadas a ampliar a autonomia pessoal e social dos usuários, ou no desenvolvimento de iniciativas de ajuda mútua (ações exemplificadas nas experiências brasileiras relatadas neste artigo). Trata-se igualmente de participar na construção de ambientes científicos compartilhados e nas estratégias de formação que permitam, efetivamente, aos usuários de serviços de saúde mental desenvolver projetos de pesquisa e produção de conhecimento.

A Reforma Psiquiátrica Brasileira tem uma forte tradição participativa de usuários e familiares nos movimentos sociais de defesa de direitos e no controle social das políticas públicas. Pode-se afirmar que a participação política dos usuários é um traço constitutivo e essencial da Reforma brasileira, e que ela se amplia para um intenso movimento cultural e para intervenções estratégicas de produção de autonomia, como as experiências de saúde mental e economia solidária. A partir dos anos 2000, iniciativas claramente articuladas a experiências internacionais que se descrevem sob as referências conceituais de empoderamento e recovery, como as relatadas neste artigo, começam a se desenvolver. É um passo adiante na participação de usuários, que abre caminho para as iniciativas de formação e produção de conhecimento com protagonismo destes. No cenário brasileiro, ainda é incipiente esta forma de participação. Para o mundo acadêmico da saúde mental no país, aos usuários e familiares cabe apenas o papel de "sujeitos de pesquisa", mesmo que inseridos em projetos de intervenção com alto potencial de produção de autonomia e empoderamento. O usuário-pesquisador começa a se fazer presente, entretanto ainda de modo pouco sistemático, nos desafios suscitados pelas experiências brasileiras aqui relatadas.

\section{Considerações Finais}

A participação de usuários e familiares na produção de conhecimento é ainda incipiente em nosso país. Não se estabeleceu até o momento uma agenda sistemática na política pública, que inclua o compartilhamento dos saberes e o protagonismo de usuários como autores do conhecimento produzido na atenção psicossocial. Entretanto, algumas experiências, tanto de pesquisas avaliativas como de intervenção, têm demonstrado as potencialidades da participação efetiva de usuários e familiares como pesquisadores. Este é um desafio relevante, não apenas para o estágio atual da reforma psiquiátrica brasileira, mas também para a ampliação das perspectivas analíticas e para o alcance social da pesquisa em psiquiatria e saúde mental no país.

\section{Colaboradores}

RF Presoto, EM Vasconcelos, PGG Delgado e M Silveira participaram igualmente de todas as etapas de elaboração do artigo. 


\section{Referências}

1. Vasconcelos EM. Empoderamento de usuários e familiares em saúde mental e em pesquisa avaliativa/interventiva: uma breve comparação entre a tradição anglo-saxônica e a experiência brasileira. Cien Saude Colet 2013; 18(10):2825-2835.

2. Slade M. Recovery research: new answers, new questions. Presentation at the Special Symposium Recovery-oriented practices. $11^{\text {th }}$ World Congress of the World Association for Psychosocial Rehabilitation. Milan, Italy, 10-13 November 2012 (anotação de um dos autores, 12/11/2012).

3. Slade M, Amering M, Oades L. Recovery: an international perspective. Epidemiol Psichiatr Soc 2008; 17(2):128-137.

4. Farkas M, Anthony WA. Psychiatric rehabilitation interventions: a review. Int Rev Psychiatry 2010; 22(2):114-129.

5. Simpson EL, House AO. Involving users in the delivery and evaluation of mental health services: systematic review. BMJ 2002; 325(7375):1265.

6. Thornicroft G, Tansella M. Growing recognition of the importance of service user involvement in mental health service planning and evaluation. Epidemiol Psichiatr Soc 2005; 14(1):1-3.

7. Chinman MJ, Rosenheck R, Lam JA, Davidson L. Comparing consumer and non consumer provided case management for homeless persons with serious mental illness. J Nerv Ment Dis 2000; 188(7): 446-453.

8. Goldstrom ID, Campbell J, Rogers JA, Lambert DB, Blacklow B, Henderson MJ, Manderscheid RW. National estimates for mental health mutual support groups, self-help organizations, and consumer-operated services. Adm Policy Ment Health 2006; 33(1):92-103.

9. Boote J, Telford R, Cooper C. Consumer involvement in health research: a review and research agenda. Health Policy 2002; 61(2):213-236.

10. Serpa Júnior OD, Leal EM, Louzada R, Silva Filho JF. A inclusão da subjetividade no ensino da psicopatologia. Interface (Botucatu) 2007; 11(22):207-222.

11. Telford R, Boote JD, Cooper CL. What does it mean to involve consumers successfully in NHS research? A consensus study. Health Expect 2004; 7(3):209-220.

12. Brasil. Ministério da Saúde. Conselho Nacional de Saúde. Resolução nº 196 de 10 de outubro de 1996. Diretrizes e Normas Regulamentadoras de Pesquisas Envolvendo Seres Humanos. Diário Oficial da União 1996; 16 out.

13. Consumers Health Forum of Australia (CHF). [página na Internet]. [acessado 2013 fev 10]. Disponível em: https://www.chf.org.au

14. Vasconcelos EM. Dispositivos associativos de luta e empoderamento de usuários, familiares e trabalhadores em saúde mental no Brasil. Vivência 2007, 32:173-206

15. Vasconcelos EM, Paes Leme CCC, Weingarten R, Novaes PR, organizadores. Reinventando a vida: narrativas de recuperação e convivência com o transtorno mental. São Paulo, Rio de Janeiro: Hucitec, EncantArte; 2006.
16. Brasil. Ministério da Saúde (MS), Universidade Federal do Rio de Janeiro. Cartilha de ajuda e suporte mútuos em saúde mental. Brasília, Rio de Janeiro: MS, UFRJ; 2013.

17. Vasconcelos EM. Complexidade e pesquisa interdisciplinar. Petrópolis: Vozes; 2002.

18. Delgado PG, Monnerat S, Dahl C, Ribeiro M, Solis F, Moraes A, Bezerra T, Couto MC. Familiares Parceiros do Cuidado. Relatório Preliminar de Intervenção e Pesquisa. Rio de Janeiro: UFRJ, Instituto de Psiquiatria, NUPPSAM; 2012.

19. Guia Pessoal de Gestão Autônoma da Medicação. [Acessado em 2013 maio 24]. Disponível em: http:/ /www.fcm.unicamp.br/interfaces/arquivos/ ggamBr.pdf

20. Onocko Campos RT, Passos E, Leal E, Serpa O. Pesquisa avaliativa de saúde mental: instrumentos para a qualificação da utilização de psicofármacos e formação de recursos humanos. Edital do CNPQ, 2009.

21. Santos DVD. Uso de psicotrópicos na atenção primária no Distrito Sudoeste de Campinas e sua relação com os arranjos da clínica ampliada [dissertação]. Campinas (SP): FCM, Unicamp; 2009.

22. Vedia UC, Bonet MS, Forcada VC, Parellada EN. Study of use of psychiatric drugs in primary care. Aten Primaria 2005; 36(5):239-245.

23. International Narcotics Control Board (INCB). Report of the INCB for 2004. [página na Internet]. [acessado 2013 jul 25]. Disponível em: http://www. incb.org/incb/en/publications/annual-reports/annual-report-2004.html

24. Hull SA, Aquino P, Cotter S. Explaining variation in antidepressant prescribing rates in east London: a cross sectional study. Fam Pract 2005; 22(1):37-42

25. Cadilhe S. Benzodiazepinas: prevalência de prescrição e concordância com os motivos de consumo. Rev Port. Clin Geral 2004; 20(2):193-202.

26. Emerich BF. Direitos dos usuários em intenso sofrimento psíquico, na perspectiva dos usuários e gestores de CAPS [dissertação]. Campinas (SP): FCM, Unicamp; 2012.

27. Presotto RF. Participação de Usuários de Serviços de Saúde Mental em Pesquisas: Um Olhar a partir dos conceitos de Empowerment e Recovery [dissertação]. Campinas (SP): FCM, Unicamp; 2013.

Artigo apresentado em 25/03/2013

Aprovado em 06/06/2013

Versão final apresentada em 21/06/2013 